\title{
Radionuclide imaging of subendocardial ischaemia: an insight into coronary pathophysiology or a technical artefact?
}

\author{
Gianmario Sambuceti $^{1,2} \cdot$ Silvia Morbelli $^{2} \cdot$ Alessandro Bellini $^{1} \cdot$ Cecilia Marini $^{2,3}$
}

Received: 25 January 2017 / Accepted: 26 January 2017 / Published online: 8 February 2017

(C) Springer-Verlag Berlin Heidelberg 2017

In the present issue of the European Journal of Nuclear Medicine and Molecular Imaging, Sciagrà and coworkers report an investigation of the mechanisms underlying a decrease in left ventricular (LV) ejection fraction during dipyridamoleinduced coronary vasodilation in a group of patients with hypertrophic cardiomyopathy (HCM) [1]. As a major finding, transient LV dysfunction did not identify patients with more severe impairment in coronary flow reserve and maximal blood flow. Rather, it was associated with stress-induced development of a transmural gradient in myocardial perfusion characterized by a relative reduction in maximal flow capacity in the subendocardial layers.

In several aspects, this study extends the notion that selective coronary vasodilation can induce myocardial ischaemia causing true subendocardial hypoperfusion even in the absence of both changes in myocardial oxygen need and significant obstructions in major coronary arteries. Concurrently, it confirms that the ability to delineate the transmural extent of perfusional, metabolic or contractile impairment might be a prerequisite for a deeper understanding of the pathophysiological mechanisms underlying a variety of cardiac disorders.

This Editorial Commentary refers to the article http://dx.doi.org/10.1007/ s00259-016-3603-2

Gianmario Sambuceti

Sambuceti@unige.it

1 Nuclear Medicine, Department of Health Sciences, University of Genoa, Genova, Italy

2 IRCCS San Martino - IST, Genova, Italy

3 CNR Institute for Bioimages and Molecular Physiology, Milan, Italy
For obvious reasons, this issue represents a specific challenge for cardiac imaging. In the setting of nuclear medicine, many studies have actually shown a relative subendocardial underperfusion under different physiological conditions. However, an accurate description of transmural flow distribution is still challenging for virtually all imaging modalities because cardiac contraction cyclically modifies the shape and thickness of the LV myocardium while respiratory activity periodically shifts the heart position throughout the time needed to measure myocardial tracer concentration. Below, we briefly discuss how these basic features of cardiac physiology affect radionuclide imaging of transmural perfusion gradient.

\section{Pathophysiology of transmural perfusion gradient}

Since the early 1970s, a large literature has documented the non-uniform distribution of blood flow across the LV wall [2, 3]. With respect to the subepicardium, the subendocardial layers are more vulnerable to ischaemia regardless of its pathophysiological mechanism, i.e. a sudden primary reduction in blood flow or an inadequate flow response to an increase in oxygen demand. In both experimental models and patients, the necrotic area associated with acute myocardial infarction damage is usually larger in the subendocardium [2] while exercise induced ischaemia is indicated by ST segment depression on exercise ECG [4].

Although some studies have shown a relatively higher oxygen need in the inner myocardial layers [2], current models explain the enhanced susceptibility in the endocardium as the result of compression by extravascular forces. This interpretation predicts that LV and intramyocardial pressures can collapse the inner capillaries and venules during systole and early diastole. According to the so-called "waterfall mechanism" [5], this compression stops local blood flow until the vessel 
reopens. Under physiological conditions, the flow debt caused by this transient occlusion is recovered in later diastolic phases despite the lower input pressure at this stage. However, conditions such as myocardial hypertrophy that enhance the extravascular compressive forces and simultaneously decrease microvessel density can markedly impair this flow repayment. Similarly, relative subendocardial hypoperfusion can occur when input pressure is markedly decreased by aortic regurgitation. Finally, this physiological mechanism is markedly hampered in coronary artery disease [6]. According to Poiseuille's law, any arterial stenosis imposes a pressure gradient that is directly proportional to flow. This physical property threatens subendocardial perfusion since it transiently increases flow need and thus pressure drop in late diastole when coronary pressure is at its lowest $[2,6]$.

These considerations explain why selective coronary vasodilation by dipyridamole can paradoxically induce myocardial ischaemia virtually without affecting myocardial oxygen demand. This phenomenon is a common observation in cardiac imaging laboratories when a severe reversible perfusion defect is accompanied by transient ST segment depression or is followed by transient regional dysfunction as a sign of postischaemic stunning. Under this condition, the marked drop in distal coronary pressure caused by pharmacological vasodilation is not counterbalanced by the increase in aortic pressure that usually accompanies exercise. This combination prolongs the duration of cyclic collapse in the endocardial microvasculature diverting blood flow to the "lesscompressed" subepicardial vessels, a process called "transmural steal" [7], i.e. the simultaneous coexistence of ischaemia in the subendocardium with luxury perfusion in the adjacent subepicardium.

\section{Radionuclide imaging of transmural perfusion gradient}

Radionuclide techniques are so far the gold standard method for estimating specific myocardial blood flow (i.e. the flow per unit mass of myocardium). As a surrogate in the clinical setting, specific myocardial blood flow is most often evaluated by determining the flow distribution throughout the LV walls with ${ }^{99 \mathrm{~m}}$ Tc-labelled tracers. Nevertheless, despite their broad utilization, the spatial resolution of most SPECT approaches has so far prevented the accurate measurement of transmural flow gradient. In contrast, the nominal spatial resolution of most PET scanners $(4-5 \mathrm{~mm})$ apparently seems to be suited to this purpose in normal walls (averaging $9-10 \mathrm{~mm}$ in enddiastole). However, this parameter defines the so-called "fullwidth at half-maximum" (FWHM), which implies that radioactivity concentration at any given point in space can only be correctly measured when the source diameter is larger than twice the FWHM. As a consequence, correctly estimating true counts in the outer and inner myocardial layers requires wall thicknesses close to $16-20 \mathrm{~mm}$.

In agreement with this concept, the first studies testing transmural perfusion gradient most intensively investigated severe LV hypertrophy and HCM [8]. Subsequently, improvements in scanner technology allowed this evaluation to be extended to nonhypertrophic myocardium both in animal experiments and in patients. Overall, the available literature confirms the hypothesis that myocardial blood flow is homogeneously distributed in the different myocardial layers under resting conditions while a relative subendocardial underperfusion can occur in the presence of vasodilation induced by pharmacological interventions. This behaviour has been documented in HCM $[8,9]$, in aortic stenosis [10], in hypertension [11], in coronary artery disease [12] and, paradoxically, in normal human subjects [13]. Overall (with the exception of normal subjects), the literature agrees with the theoretical models of coronary physiology, explains the occurrence of ischaemia in HCM, and seems to confirm a significant advantage for PET in myocardial perfusion imaging. However, although the accuracy of PET in measuring blood flow in different LV wall layers has been experimentally documented [14], its ability to accurately measure transmural perfusion gradient has not been fully validated.

The introduction of labelled microspheres led to an understanding of the relevance of transmural flow gradient and its underlying mechanisms [15]. With a few exceptions [16], this technique has been limited to the experimental setting and almost always involves ex-vivo counting, without any limitation in sample size. Nevertheless, imaging myocardial perfusion in living humans has to address the need for an appropriate dosimetry with the unavoidable interference of the cardiac cycle and thus of periodical changes in ventricular volume and wall thickness. Both factors have profound implications and have to be accurately considered to correctly interpret indexes of transmural distribution of blood flow.

\section{Transmural perfusion gradient in thickening and moving myocardium with diffusible tracers}

Overall, the studies discussed above have shown a subendocardial/subepicardial flow ratio in the range 0.8 1.2 , i.e. not far from 1 . Identifying such subtle differences requires an accurate estimation of relative flow values. This requirement has different implications according to the tracer used and the kinetic model adopted in any specific study.

Due to its almost optimal kinetic features, many studies of absolute myocardial blood flow and transmural flow gradient have been performed using ${ }^{15} \mathrm{O}-\mathrm{H}_{2} \mathrm{O}$. From the imaging point of view, this tracer, however, displays several peculiarities. First, the positron emission of ${ }^{15} \mathrm{O}$ is characterized by a 
positron range in water almost $50 \%$ wider than that of the most commonly used ${ }^{18} \mathrm{~F}$ that further increases the uncertainty in spatial identification of positron emission [17]. Second, as does every diffusible tracer, water disperses within the myocardial tissue and is washed from it according to the ratio between flow and the perfused tissue. As a consequence, images can only be obtained by the set-up of parametric maps representing calculated blood flow and perfusable tissue fraction in each voxel. This computational algorithm processes series of frames lasting 5-30 s. In practice, the time needed for this process does not hamper the evaluation of arterial input function. However, it is always longer than a single cardiac cycle. As an inevitable consequence, in any given frame the radioactivity measured in all voxels of the apparent inner LV myocardial border will include an indistinguishable contribution from the tracer content in the endocardium and LV cavity. In other words, the shape of the curve measured in the inner LV wall will inevitably include a mixed and inseparable contribution of tracer concentration from the arterial blood (in diastole) and the LV wall (in systole). This confounding effect is obviously reversed in the outer borders of the LV image, with the subepicardium being mostly represented in diastole and the pericardial (mediastinal) space in systole. This double interference is thus opposite in phase and only modestly hampers the measurement of transmural myocardial blood flow. However, its potential contribution can become particularly relevant in the evaluation of transmural flow gradient that can be markedly affected by the criteria used to define the limits of the apparent myocardial wall. Finally, this task is further threatened by the notion that the amount of tissue eventually reached by perfusion can markedly change in response to changes in perfusion pressure, thus limiting the adherence of specific blood flow with the actual flow need [18].

\section{Transmural perfusion gradient in thickening and moving myocardium with deposit tracers}

Differently from water, deposit tracers such as ${ }^{13} \mathrm{~N}$-ammonia [8], ${ }^{82} \mathrm{Rb}$ [19] and ${ }^{18}$ F-flurpiridaz [20] are characterized by a relatively stable bond with the reached cardiomyocytes and by a washout rate slow enough not to interfere with imaging. Overall, this kinetic feature indicates that tracer uptake is a function of local blood flow that can be estimated by dedicated compartmental models able to describe the rate constant of tracer exchange between arterial blood and tissue during the first minutes after tracer injection. As a consequence, timeconcentration curve in the different myocardial layers is obviously affected by cyclic cardiac movement without significant differences with respect to the analysis of water kinetics.

On theoretical grounds, the use of ${ }^{13} \mathrm{~N}$-ammonia might overcome this limitation. Differently from compartmental analysis, the so-called "fist-pass extraction" method [21] does not process the entire tracer concentration curve within the myocardial tissue. Rather, it stochastically estimates myocardial blood flow by normalizing late myocardial tracer concentration to the integral of estimated arterial input function. In many aspects, this approach thus combines the advantages offered by PET detection and the user-friendly procedure commonly used with ${ }^{99 \mathrm{~m}}$ Tc labelled perfusion tracers permitting a delay in imaging when baseline haemodynamics is restored. Nevertheless, despite the high resolution and the optimal counting statistics, the analysis of the transmural gradient in these perfusion images remains flawed by the interference of cardiac contraction, for the following reasons.

Regardless of the isotope used, myocardial perfusion imaging involves the measurement of the gamma emission rate. In other words, signal intensity (the index of myocardial blood flow) is the number of recorded photons. Assimilating this procedure to photography, the intensity of reproduced light represents the product of the photon emission rate and the time used for its recording. The diagram shown in Fig. 1 illustrates this concept. Using the same exposure time ( $1 \mathrm{~s})$ the lights of a standing car appear markedly brighter than the nearby street lamp (Fig. 1a). The difference is reduced if the car moves slowly (Fig. 1b) and is inverted at high speed (Fig. 1c). This phenomenon occurs simply because interaction between photons emitted by the car lights and any given point on the photographic film (or matrix) is inversely correlated with the car speed while the photons emitted by the standing lamp will hit the same film "pixel" for the whole exposure time.

Extending this daily experience to the technical aspects of myocardial perfusion imaging, one should bear in mind that heart rate and cardiac function are different in different patients or in the same patient under different pathophysiological conditions. These differences apply to both harmonic parameters of cyclic cardiac contraction: phase and amplitude. In the absence of arrhythmias, phase variability is related to the basic concept of cardiac physiology in which systolic time is considered to be relatively fixed. By contrast, increases in heart rate shorten the time available for diastole and the shape of its curve, mostly by prolonging the duration of the last phase when LV volume is close to its maximal value. As a consequence, the appearance of the LV provided by an acquisition of 5-10 $\mathrm{min}$ is dependent upon heart rate. To explain this concept, Fig. 2 is a schematic representation of the short axis of a LV with the same end-diastolic diameter and without any transmural perfusion gradient. Figure $2 \mathrm{a}$ shows the eight frames acquired throughout an R-R interval at a heart rate of $90 \mathrm{bpm}$. Under these conditions the $\mathrm{T}$ wave and end-systole occur roughly in the middle of the sequence (i.e. between frame 4 and 5). Apparent flow distribution in the ungated image will be relatively homogeneous, although with slightly higher values in the endocardial layer due to the interference of partial volume effect and systolic wall thickening that increase the recovery coefficient during systole [22]. Figure $2 b$ 


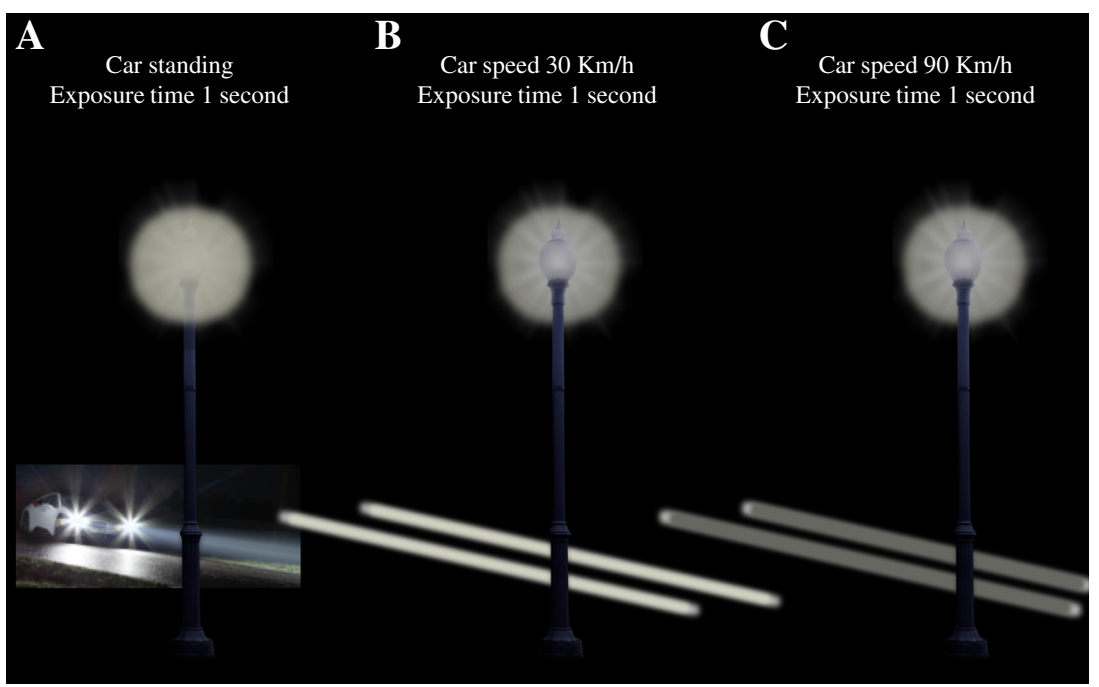

Fig. 1 Intensity of reproduced light, photon emission rate and time of recording. Using the same exposure time $(1 \mathrm{~s})$ the lights of a standing car appear markedly brighter than the nearby street lamp (a). The difference is reduced if the car moves slowly (b) and is inverted at high speed (c).

shows the profoundly different pattern observed for the same heart at a heart rate of $45 \mathrm{bpm}$. Under these conditions, the prolonged duration of late diastole increases the recovered counts in the outer LV border, apparently reversing the flow distribution in favour of the subepicardial layers.

An even more obvious shift is apparent when bradycardia is associated with a significant impairment in contractile
This phenomenon occurs because interaction between photons emitted by the car lights and any given point on the photographic film (or matrix) is inversely correlated with the car speed while the photons emitted by the standing lamp will hit the same film "pixel" for the whole exposure time

function. Under these conditions, the effects of a long late diastole are enhanced by the loss of relative endocardial overestimation due to the reduction in wall motion and, mostly, in wall thickening. This combination inevitably results in a marked underestimation of blood flow in the inner LV layers, as shown in Fig. 2c. Since myocardial dysfunction can be either chronic or transient, the contribution of this technical

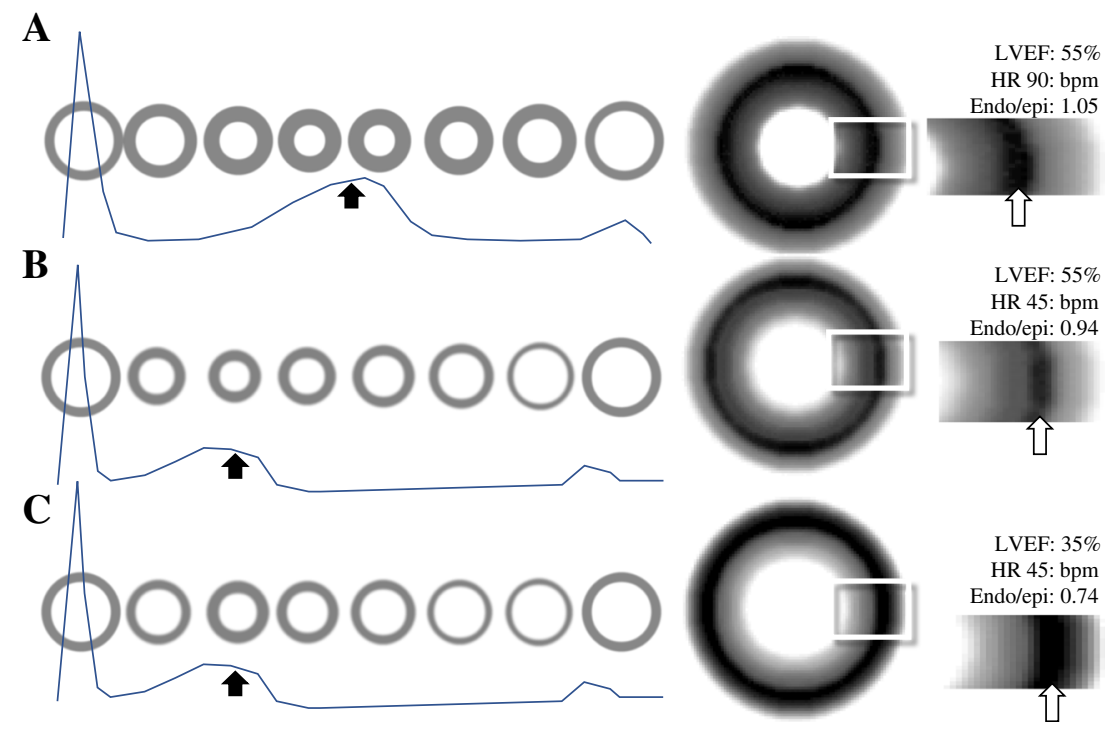

Fig. 2 Schematic representation of the short axis of a left ventricle with the same end-diastolic diameter and without any transmural perfusion gradient. a The eight frames acquired throughout an R-R interval at a heart rate of $90 \mathrm{bpm}$. The apparent flow distribution in the ungated image will be relatively homogeneous although with slightly higher values in the endocardial layer due to the interference of the partial volume effect and systolic wall thickening that increase the recovery coefficient during systole. b A profoundly different pattern is observed for the same heart at a heart rate of $45 \mathrm{bpm}$. Under these conditions, the prolonged duration of late diastole increases the recovered counts in the outer LV border, apparently reversing the flow distribution in favour of the subepicardial layers. c An even more obvious shift is apparent when bradycardia is associated with a significant impairment in contractile function. Under these conditions, the effects of a long late diastole are enhanced by the loss of relative endocardial overestimation due to the reduction in wall motion and, mostly, in wall thickening. This combination inevitably results in a marked underestimation of blood flow in the inner LV layers 
pitfall to the apparent subepicardial shift of blood flow should be carefully considered during transient ischaemia or transient dysfunction whatever the cause.

\section{Conclusions}

The harmonic nature of heart function inevitably implies a significant difference in flow regulation throughout the different LV myocardial layers. The ability to recognize the increased susceptibility of the subendocardial layers might surely enhance the accuracy of methods focused on the detection of ischaemia. Radionuclide methods have this potential. However, the combination of limited spatial resolution and the need for adequate counting statistics are still difficult challenges. In this regard, the possibility to depict LV wall position during the cardiac cycle might be a major step forward. ECG gating might be at least a partial solution to this problem due to its ability to describe the position and thickness of the LV wall in the different systolic and diastolic phases. However, ECG gating during the phase of tracer exchange between blood and tissue is extremely difficult. Verifying the performance of this "stroboscopic" synchronization in the analysis of late steadystate images would surely represent a first step in the right direction.

Compliance with ethical standards This article does not describe any studies with human participants or animals performed by any of the authors.

Conflicts of interest None.

\section{References}

1. Sciagrà R, Calabretta R, Cipollini F, Passeri A, Castello A, Cecchi F, et al. Myocardial blood flow and left ventricular functional reserve in hypertrophic cardiomyopathy: a 13NH3 gated PET study. Eur J Nucl Med Mol Imaging. 2017. doi:10.1007/s00259-0163603-2.

2. Hoffman JI. Transmural myocardial perfusion. Prog Cardiovasc Dis. 1987;29:429-464.

3. Rubio R, Berne RM. Regulation of coronary blood flow. Prog Cardiovasc Dis. 1975;18:105-122.

4. Ross Jr J. Electrocardiographic ST-segment analysis in the characterization of myocardial ischemia and infarction. Circulation. 1976;53(3 Suppl):I73-I81.

5. Downey JM, Kirk ES. Inhibition of coronary blood flow by a vascular waterfall mechanism. Circ Res. 1975;36:753-760.

6. Hoffman JI, Spaan JA. Pressure-flow relations in coronary circulation. Physiol Rev. 1990;70:331-390.

7. Meerdink DJ, Okada RD, Leppo JA. The effect of dipyridamole on transmural blood flow gradients. Chest. 1989;96:400-405.

8. Gistri R, Cecchi F, Choudhury L, Montereggi A, Sorace O, Salvadori PA, et al. Effect of verapamil treatment on absolute myocardial blood flow in hypertrophic cardiomyopathy. Am J Cardiol. 1994;74:363-368.

9. Knaapen P, Germans T, Camici PG, Rimoldi OE, ten Cate FJ, ten Berg JM, et al. Determinants of coronary microvascular dysfunction in symptomatic hypertrophic cardiomyopathy. Am J Physiol Heart Circ Physiol. 2008;294(2):H986-93.

10. Rajappan K, Rimoldi OE, Dutka DP, Ariff B, Pennell DJ, Sheridan DJ, et al. Mechanisms of coronary microcirculatory dysfunction in patients with aortic stenosis and angiographically normal coronary arteries. Circulation. 2002;105(4):470-476.

11. Rimoldi O, Rosen SD, Camici PG. The blunting of coronary flow reserve in hypertension with left ventricular hypertrophy is transmural and correlates with systolic blood pressure. J Hypertens. 2014;32(12):2465-2471. doi:10.1097/HJH. 0000000000000338.

12. Danad I, Raijmakers PG, Harms HJ, Heymans MW, van Royen N, Lubberink M, et al. Impact of anatomical and functional severity of coronary atherosclerotic plaques on the transmural perfusion gradient: a [15O]H2O PET study. Eur Heart J. 2014;35(31):2094-2105. doi:10.1093/eurheartj/ehu170.

13. Vermeltfoort IA, Raijmakers PG, Lubberink M, Germans T, van Rossum AC, Lammertsma AA, et al. Feasibility of subendocardial and subepicardial myocardial perfusion measurements in healthy normals with (15)O-labeled water and positron emission tomography. J Nucl Cardiol. 2011;18:650-656.

14. Rimoldi O, Schafers KP, Boellaard R, Turkheimer F, Stegger L, Law MP, et al. Quantification of subendocardial and subepicardial blood flow using 15O-labeled water and PET: experimental validation. J Nucl Med. 2006;47:163-172.

15. Heymann MA, Payne BD, Hoffman JI, Rudolph AM. Blood flow measurements with radionuclide-labeled particles. Prog Cardiovasc Dis. 1977;20(1):55-79.

16. Parodi O, Sambuceti G, Roghi A, Testa R, Inglese E, Pirelli S, et al. Residual coronary reserve despite decreased resting blood flow in patients with critical coronary lesions. A study by technetium- $99 \mathrm{~m}$ human albumin microsphere myocardial scintigraphy. Circulation. 1993;87:330-344.

17. Levin GS, Hoffman EJ. Calculation of positron range and its effect on the fundamental limit of positron emission tomography system spatial resolution. Phys Med Biol. 1999;44:781-799.

18. Sambuceti G, Marzilli M, Mari A, Marini C, Schluter M, Testa R, et al. Coronary microcirculatory vasoconstriction is heterogeneously distributed in acutely ischemic myocardium. Am J Physiol Heart Circ Physiol. 2005;288:H2298-H2305.

19. Lautamäki R, George RT, Kitagawa K, Higuchi T, Merrill J, Voicu C, et al. Rubidium-82 PET-CT for quantitative assessment of myocardial blood flow: validation in a canine model of coronary artery stenosis. Eur J Nucl Med Mol Imaging. 2009;36:576-586. doi:10. 1007/s00259-008-0972-1.

20. Sherif HM, Nekolla SG, Saraste A, Reder S, Yu M, Robinson S, et al. Simplified quantification of myocardial flow reserve with flurpiridaz F18: validation with microspheres in a pig model. J Nucl Med. 2011;52:617-624. doi:10.2967/jnumed.110.083196.

21. Bellina CR, Parodi O, Camici P, Salvadori PA, Taddei L, Fusani L, et al. Simultaneous in vitro and in vivo validation of nitrogen-13ammonia for the assessment of regional myocardial blood flow. J Nucl Med. 1990;31:1335-1343.

22. Marcassa C, Marzullo P, Parodi O, Sambuceti G, L'Abbate A. A new method for noninvasive quantitation of segmental myocardial wall thickening using technetium-99m 2-methoxy-isobutylisonitrile scintigraphy - results in normal subjects. J Nucl Med. 1990;31:173-177. 